\title{
Spectral formation in accreting X-ray pulsars: bimodal variation of the cyclotron energy with luminosity
}

\author{
P. A. Becker ${ }^{1}$, D. Klochkov ${ }^{2}$, G. Schönherr ${ }^{3}$, O. Nishimura ${ }^{4}$, C. Ferrigno ${ }^{5}$, I. Caballero ${ }^{6}$, P. Kretschmar ${ }^{7}$, M. T. Wolff ${ }^{8}$, \\ J. Wilms ${ }^{9}$, and R. Staubert ${ }^{2}$
}

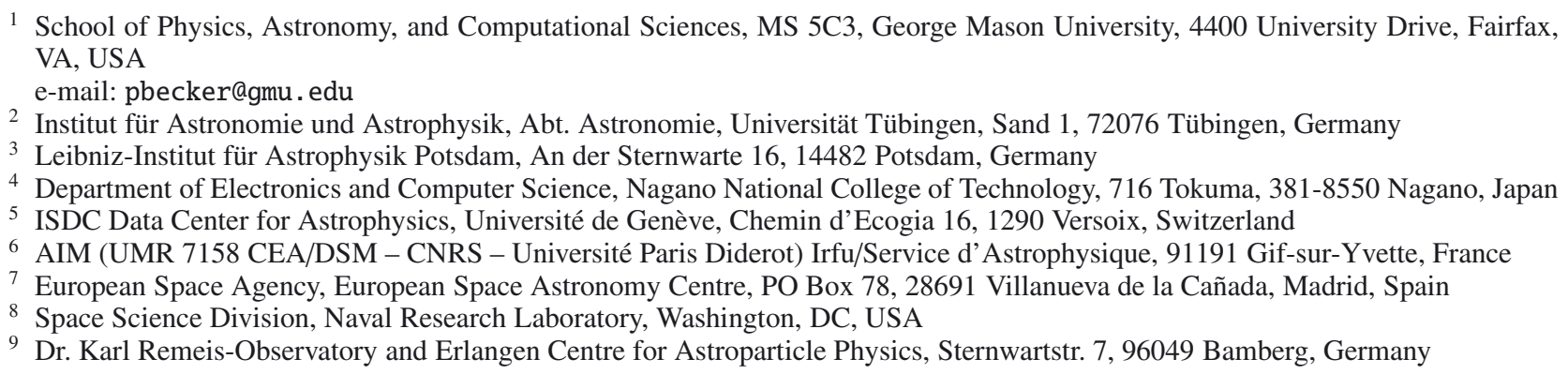

Received 17 February 2012 / Accepted 22 May 2012

\begin{abstract}
Context. Accretion-powered X-ray pulsars exhibit significant variability of the cyclotron resonance scattering feature (CRSF) centroid energy on pulse-to-pulse timescales, and also on much longer timescales. Two types of spectral variability are observed. For sources in group 1, the CRSF energy is negatively correlated with the variable source luminosity, and for sources in group 2, the opposite behavior is observed. The physical basis for this bimodal behavior is currently not well understood.

Aims. We explore the hypothesis that the accretion dynamics in the group 1 sources is dominated by radiation pressure near the stellar surface, and that Coulomb interactions decelerate the gas to rest in the group 2 sources.

Methods. We derive a new expression for the critical luminosity, $L_{\text {crit }}$, such that radiation pressure decelerates the matter to rest in sources with X-ray luminosity $L_{\mathrm{X}}>L_{\text {crit }}$. The formula for $L_{\text {crit }}$ is based on a simple physical model for the structure of the accretion column in luminous X-ray pulsars that takes into account radiative deceleration, the energy dependence of the cyclotron cross section, the thermodynamics of the accreting gas, the dipole structure of the pulsar magnetosphere, and the diffusive escape of radiation through the column walls. We show that for typical neutron star parameters, $L_{\text {crit }}=1.5 \times 10^{37} B_{12}^{16 / 15} \mathrm{erg} \mathrm{s}^{-1}$, where $B_{12}$ is the surface magnetic field strength in units of $10^{12} \mathrm{G}$.

Results. The formula for the critical luminosity is evaluated for five sources, using the maximum value of the CRSF centroid energy to estimate the surface magnetic field strength $B_{12}$. The results confirm that the group 1 sources are supercritical $\left(L_{\mathrm{X}}>L_{\text {crit }}\right)$ and the group 2 sources are subcritical $\left(L_{\mathrm{X}}<L_{\text {crit }}\right)$, although the situation is less clear for those highly variable sources that cross over the line $L_{\mathrm{X}}=L_{\text {crit }}$. We also explain the variation of the CRSF energy with luminosity as a consequence of the variation of the characteristic emission height. The sign of this dependence is opposite in the supercritical and subcritical cases, hence creating the observed bimodal behavior.

Conclusions. We have developed a new model for the critical luminosity in accretion-powered X-ray pulsars that explains the bimodal dependence of the CRSF centroid energy on the X-ray luminosity $L_{X}$. Our model provides a physical basis for the observed variation of the CRSF energy as a function of $L_{\mathrm{X}}$ for both the group 1 (supercritical) and the group 2 (subcritical) sources as a result of the variation of the emission height in the column.
\end{abstract}

Key words. stars: neutron - pulsars: general - radiative transfer - accretion, accretion disks

\section{Introduction}

X-ray binary pulsars (XRBPs) were first observed by Giacconi et al. (1971) and Tananbaum et al. (1972), and now include many of the brightest sources in the X-ray sky. In XRBPs, the main sequence companion star transfers matter to the neutron star via Roche lobe overflow, or via a strong stellar wind (Frank et al. 2002). The gas forms an accretion disk around the neutron star, and the material spirals inward until the pressure of the star's dipole magnetic field becomes comparable to the ram pressure of the matter in the disk. This occurs at the Alfvén radius, located several thousand kilometers out in the accretion disk.
The fully-ionized accreting plasma is entrained by the magnetic field at the Alfvén radius, and from there the matter is guided through the magnetosphere, forming accretion columns at one or both of the magnetic poles of the star. As the star spins, the inclination angle between the star's magnetic axis and the axis of the accretion disk changes, and therefore the Alfvén radius varies with the spin period of the star.

The observed X-ray emission is powered by the conversion of gravitational potential energy into kinetic energy, which is then transferred to the radiation field via electron scattering, and ultimately escapes through the walls of the column. The structure of the accretion column is maintained by the 

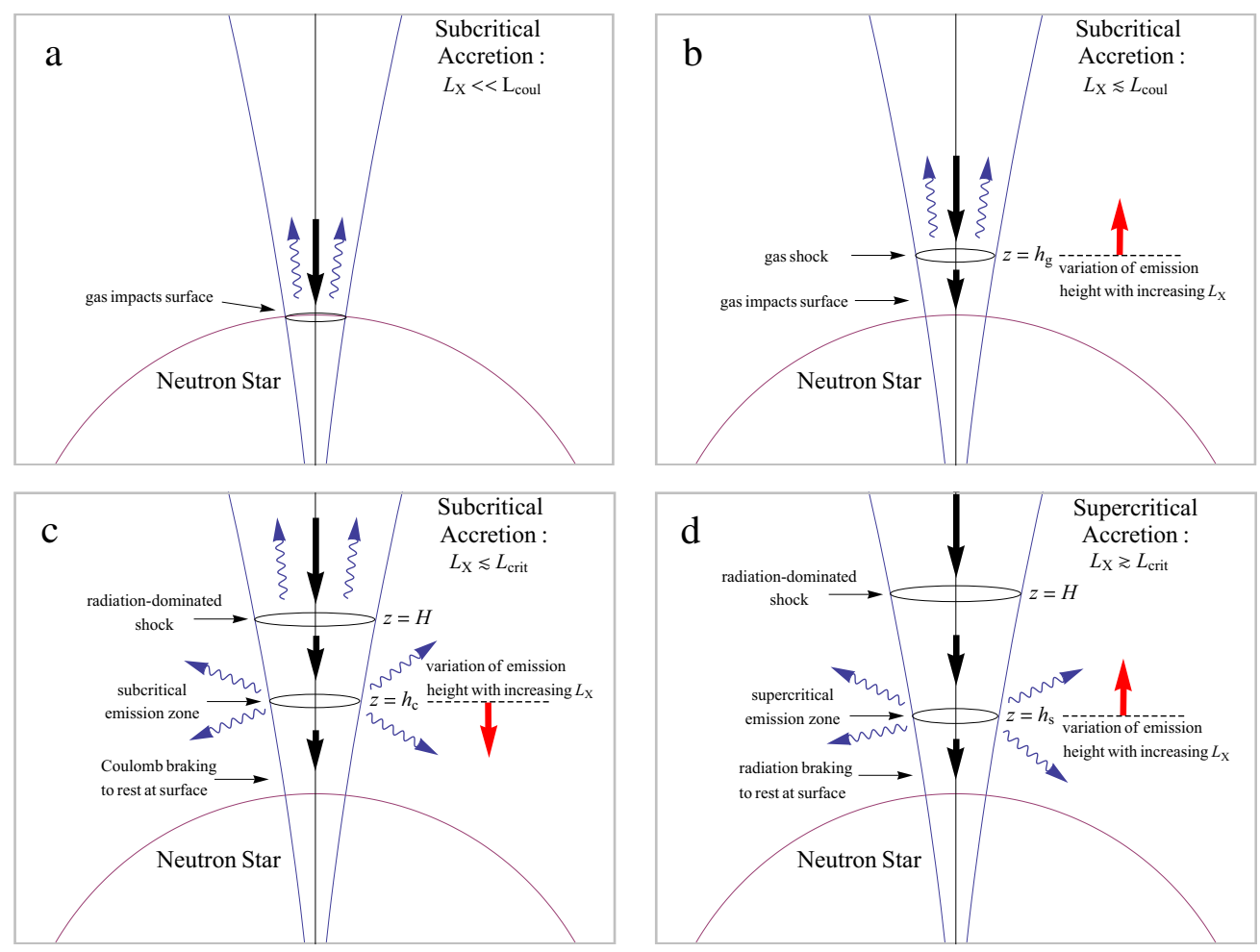

Fig. 1. Schematic illustration of the geometry of the accretion column and the variation of the characteristic emission height and emission beam pattern with increasing luminosity $L_{\mathrm{X}}$ : a) subcritical, $L_{\mathrm{X}}<L_{\text {coul }}<L_{\text {crit }}$, pencil beam; b) subcritical, $L_{\mathrm{X}} \lesssim L_{\text {coul }}<L_{\text {crit }}$, pencil beam; c) subcritical, $L_{\text {coul }}<L_{X} \lesssim L_{\text {crit }}$, intermediate beam pattern; d) supercritical, $L_{X} \gtrsim L_{\text {crit }}$, fan beam.

strong magnetic field, with a surface strength $B_{*} \gtrsim 10^{12} \mathrm{G}$, which results in a magnetic pressure far exceeding that of either the gas or the radiation field. The high incident speed of the freely-falling plasma, $\sim 0.6 c$, creates very high temperatures, $T \sim 10^{8} \mathrm{~K}$. However, the observed $\mathrm{X}$-ray pulsar spectra are highly nonthermal, indicating that the accreting gas is unable to equilibrate during the accretion timescale. In this situation, bulk and thermal Comptonization play key roles in establishing the shape of the observed spectra (Becker \& Wolff 2007).

The X-ray spectra of many XRBPs contain cyclotron resonant scattering features (CRSFs) appearing as absorption lines. The features are caused by resonant scattering of photons off plasma electrons whose energy is quantized according to their Landau level (see e.g. Trümper et al. 1978; Isenberg et al. 1998; Araya-Góchez \& Harding 2000). The CRSFs, when detected, provide a direct measurement of the magnetic field strength at the characteristic altitude of the X-ray emission. The energy of the fundamental line and the spacing between the harmonics are approximately proportional to the $B$-field strength.

Many XRBPs display X-ray spectra that vary significantly with luminosity on timescales much longer than the pulsation period. In particular, variations in the energy of CRSFs as a function of luminosity on timescales of days to months have been detected in V 0332+53 (Mowlavi et al. 2006; Tsygankov et al. 2010), 4U 0115+63 (Mihara et al. 2004; Tsygankov et al. 2007), and Her X-1 (Staubert et al. 2007; Vasco et al. 2011). In addition to the longer-term variability, there is also mounting evidence for pulse-to-pulse variability, in which the spectral hardness, the centroid energy of the CRSF, and the luminosity vary in a correlated way (Klochkov et al. 2011). This short-timescale variability is likely related to the non-stationarity of the accretion flow, perhaps indicating that the entrainment of matter from the disk onto the magnetic field lines results in filaments and blobs of accreting gas which are then channeled onto the star in a non-uniform way.

The data from both long-term and short-term (pulse-topulse) observations point to the existence of two types/modes of spectral variability (see discussion by Klochkov et al. 2011). For sources in group 1 (e.g., V 0332+53), the centroid energy of the CRSF is negatively correlated with luminosity. For sources in group 2 (e.g., Her X-1), the opposite behavior is observed. The type of spectral variability is likely driven by the mode of accretion, which in turn is determined by the luminosity (see discussion in Staubert et al. 2007). Staubert et al. (2007) and Klochkov et al. (2011) have proposed that for sources in group 1, the deceleration of the flow to rest at the stellar surface is accomplished by the pressure of the radiation field, and in the group 2 sources the deceleration occurs via Coulomb interactions. In this interpretation, a given source falls in one group or the other depending on the value of its $\mathrm{X}$-ray luminosity, $L_{\mathrm{X}}$, relative to the critical luminosity, $L_{\text {crit }}$. The hypothesis is that the group 1 sources are supercritical, with X-ray luminosity $L_{X}>L_{\text {crit }}$, and the group 2 sources are subcritical $\left(L_{\mathrm{X}}<L_{\text {crit }}\right)$.

The theory predicts that sources in their supercritical state should display a negative correlation between the luminosity and the cyclotron energy, while sources in the subcritical state should display the reverse behavior. Geometrically, the variation of the CRSF energy with luminosity is connected with variation of the characteristic emission height, which is the altitude in the accretion column where the cyclotron absorption feature is imprinted on the observed spectrum. The variation of the emission height as a function of luminosity in the subcritical and supercritical cases is indicated schematically in Fig. 1.

The general picture described above provides a qualitative basis for the interpretation of the observed correlated variation of the CRSF centroid energy with X-ray luminosity in 
some XRBPs. However, in order to obtain a quantitative understanding of these observations, one must develop a more detailed physical model for the critical luminosity, and for the dependence of the CRSF energy on the luminosity in the subcritical and supercritical regimes. The first goal of this paper is to derive a new expression for the critical luminosity, taking into account the magnetospheric connection between the radius of the accretion column and the Alfvén radius in the disk, and the energy and angle dependence of the cyclotron scattering cross section. The second goal is to examine the dependence of the CRSF centroid energy on the luminosity in the subcritical and supercritical sources.

The remainder of the paper is organized as follows. In Sect. 2, we obtain a fundamental expression for the critical luminosity that depends on the stellar mass, radius, and surface magnetic field strength. In Sect. 3 we develop simple physical models for the variation of the characteristic emission height as a function of the luminosity for subcritical and supercritical sources. In Sect. 4, we evaluate the critical luminosity based on measurements of the CRSF centroid energy for several XRBPs. We use our subcritical and supercritical models for the variation of the emission height to predict the variation of the CRSF energy as a function of luminosity. The predicted spectral variability is compared with the observational data for each source. We discuss our results and draw conclusions in Sect. 5.

\section{Critical luminosity}

\subsection{Eddington luminosity and radiative deceleration}

First we recall the definition of the standard Eddington luminosity, $L_{\text {Edd }}$, for spherically symmetric accretion onto a central mass $M_{*}$. If the accreting gas is fully-ionized hydrogen, we obtain

$L_{\mathrm{Edd}}=\frac{4 \pi G M_{*} m_{\mathrm{p}} c}{\sigma_{\mathrm{T}}}$,

where $\sigma_{\mathrm{T}}$ is the Thomson cross section, $m_{\mathrm{p}}$ is the proton mass, $c$ is the speed of light, and $G$ is the gravitational constant. When the X-ray luminosity $L_{\mathrm{X}}=L_{\mathrm{Edd}}$, the rate at which momentum is transferred to the gas via Compton scattering balances the gravitational force. Hence if $L_{\mathrm{X}}>L_{\mathrm{Edd}}$, the net force is in the outward direction and the gas decelerates as it falls toward the central mass.

We need to make two adjustments to Eq. (1) in order to compute the effective Eddington limit, $L_{\mathrm{Edd}}^{*}$, appropriate for treating $\mathrm{X}$-ray pulsar accretion columns. The first adjustment is to replace the Thomson cross section $\sigma_{\mathrm{T}}$ with $\sigma_{\|}$, which represents the mean scattering cross section for photons propagating parallel to the magnetic field axis. The second adjustment is to take the geometry of the accretion flow into account by reducing the luminosity by the ratio of the column cross-sectional area divided by the surface area of the star. Employing these corrections yields for the effective Eddington limit

$L_{\mathrm{Edd}}^{*}=L_{\mathrm{Edd}} \frac{\sigma_{\mathrm{T}}}{\sigma_{\|}} \frac{\pi r_{0}^{2}}{4 \pi R_{*}^{2}}=\frac{G M_{*} m_{\mathrm{p}} c}{\sigma_{\|}} \frac{\pi r_{0}^{2}}{R_{*}^{2}}$,

where $R_{*}$ is the stellar radius and $r_{0}$ denotes the radius of the accretion column, which we assume to have an approximately cylindrical geometry.

Based on Eq. (2), Basko \& Sunyaev (1976) concluded that for X-ray luminosities $L_{\mathrm{X}} \gtrsim 10^{36} \mathrm{erg} \mathrm{s}^{-1}$, the incident, freelyfalling gas is decelerated by a vertical flux of radiation that is locally super-Eddington. The scattering of the incident radiation removes kinetic energy from the electrons (and from the protons via Coulomb coupling), thereby decelerating the gas. Although the radiation flux seen by the gas inside the column is propagating upward, the X-rays that ultimately carry away the kinetic energy actually escape through the walls of the column, rather than the top, unless the luminosity $L_{\mathrm{X}} \lesssim 10^{36} \mathrm{erg} \mathrm{s}^{-1}$.

The deceleration of the gas begins when the freely-falling material encounters a radiation-dominated shock whose height above the star increases with increasing luminosity, reaching an altitude of several kilometers for $L_{X} \sim 10^{37-38} \mathrm{erg} \mathrm{s}^{-1}$. As the gas passes through the shock, the accretion velocity is reduced by a factor of $\sim 7$. Even though the radiation flux inside the column is super-Eddington, the height of the radiation-dominated shock is stable. This reflects the fact that the shock is a wave structure, and is not composed of a fixed population of particles. Matter moves through the shock and decelerates, but the shock height remains fixed, unless the luminosity changes. Unlike a classical gas-mediated shock, the radiation-dominated shock is not discontinuous, and instead has a thickness that is a few times larger than the mean-free path for radiation scattering.

Below the radiation-dominated shock, the matter is further decelerated in the hydrostatic "sinking regime", in which the remaining momentum is transferred to the radiation field and radiated away through the column walls (Basko \& Sunyaev 1976). The specific mechanism accomplishing the final deceleration to rest at the stellar surface in the sinking region depends on the luminosity of the accretion flow (see Fig. 1). At the highest luminosities, $L_{\mathrm{X}} \sim 10^{37-38} \mathrm{erg} \mathrm{s}^{-1}$, the radiation field accomplishes the deceleration all the way down to the stellar surface (Basko \& Sunyaev 1976). At intermediate luminosities $L_{\mathrm{X}} \sim 10^{36-37} \mathrm{erg} \mathrm{s}^{-1}$, the final phase of deceleration may occur via Coulomb breaking in a plasma cloud just above the stellar surface (Nelson et al. 1993). It is expected that at very low luminosities, $L_{\mathrm{X}} \lesssim 10^{34-35} \mathrm{erg} \mathrm{s}^{-1}$, there is no radiation-dominated shock at all, and the material passes through a conventional gasmediated shock at altitude $z=h_{\mathrm{g}}$ before striking the stellar surface (Langer \& Rappaport 1982).

The angular pattern of the emitted radiation also depends on the luminosity (see Fig. 1). In high-luminosity sources $\left(L_{X} \sim 10^{37-38} \mathrm{erg} \mathrm{s}^{-1}\right)$, the emitted radiation primarily escapes through the column walls in the sinking region, forming a "fan beam" (Davidson 1973). For low-luminosity sources $\left(L_{X} \lesssim\right.$ $10^{35} \mathrm{erg} \mathrm{s}^{-1}$ ), the emission escapes from the top of the column, forming a "pencil beam" (Burnard et al. 1991; Nelson et al. 1993). In the intermediate range, $L_{X} \lesssim 10^{35-37} \mathrm{erg} \mathrm{s}^{-1}$, the emission pattern may be a hybrid combination of these two types (Blum \& Kraus 2000).

Focusing on the high-luminosity case for now, we can estimate the luminosity required to decelerate the gas to rest at the stellar surface by considering the physical processes occurring in the sinking region below the radiation-dominated shock. The accreting matter approaches the top of the shock with the incident free-fall velocity, which we approximate using the value at the stellar surface,

$v_{\mathrm{ff}}=\left(\frac{2 G M_{*}}{R_{*}}\right)^{1 / 2}$.

Advection is dominant over diffusion in the shock, and therefore very little radiation energy escapes through the walls of the accretion column in the vicinity of the shock (Burnard et al. 1991). Hence the shock jump conditions are well approximated by the standard Rankine-Hugoniot relations for a gas with adiabatic 
index $\gamma=4 / 3$ (Basko \& Sunyaev 1976). In this case, the matter leaves the shock with the post-shock velocity

$v_{\mathrm{ps}}=\frac{1}{7} v_{\mathrm{ff}}=\frac{1}{7}\left(\frac{2 G M_{*}}{R_{*}}\right)^{1 / 2}$,

where we have assumed that the shock is strong, which is reasonable in the luminous sources (Becker 1998). If the altitude of the radiation-dominated shock above the stellar surface is $H$, and the gas decelerates at a constant rate $a$ from the post-shock velocity $v_{\mathrm{ff}} / 7$ to rest at the stellar surface in the dynamical time $t_{\mathrm{dyn}}$, then we can write the simple kinematical relations

$H=\frac{1}{2} a t_{\mathrm{dyn}}^{2}, \quad v_{\mathrm{ps}}=a t_{\mathrm{dyn}}$.

Upon elimination of $t_{\mathrm{dyn}}$, we obtain for the required upward acceleration

$a=\frac{v_{\mathrm{ps}}^{2}}{2 H}=\frac{G M_{*}}{49 R_{*} H}$.

Since the effective gravity is reduced by the pressure of the radiation field, the net acceleration can also be related to the luminosity $L_{\mathrm{X}}$ via

$a=\left(\frac{L_{\mathrm{X}}}{L_{\mathrm{Edd}}^{*}}-1\right) \frac{G M_{*}}{R_{*}^{2}}$.

Setting Eqs. (6) and (7) equal and solving for $L_{X}$ yields

$L_{\mathrm{X}}=L_{\text {crit }} \equiv L_{\text {Edd }}^{*}\left(\frac{R_{*}}{49 H}+1\right)$.

Substituting for $L_{\text {Edd }}^{*}$ using Eq. (2), we obtain for the critical luminosity

$L_{\text {crit }}=\frac{G M_{*} m_{\mathrm{p}} c}{\sigma_{\|}} \frac{\pi r_{0}^{2}}{R_{*}^{2}}\left(\frac{R_{*}}{49 H}+1\right)$.

Our goal is to express the parameters $r_{0}, \sigma_{\|}$, and $H$ appearing on the right-hand side of Eq. (9) in terms of observable quantities.

\subsection{Radiation-dominated shock height}

The altitude, $H$, of the radiation-dominated shock can be estimated by considering the relationship between the dynamical timescale for deceleration, $t_{\text {dyn }}$, and the photon escape timescale, $t_{\text {diff }}$, which is the mean time it takes the photons to diffuse through the walls of the accretion column. In order for the gas to come to rest at the stellar surface, these two timescales must be comparable in the sinking region below the shock, which is a general property of accretion flows onto white dwarf stars and neutron stars (e.g., Imamura et al. 1987). Combining Eqs. (4) and (5), we obtain for the dynamical time

$t_{\mathrm{dyn}}=\frac{2 H}{v_{\mathrm{ps}}}=14 H\left(\frac{R_{*}}{2 G M_{*}}\right)^{1 / 2}$.

The escape timescale for the photons to diffuse through the column walls is estimated by writing

$t_{\mathrm{esc}}=\frac{r_{0}}{v_{\perp}^{\mathrm{diff}}}, \quad v_{\perp}^{\mathrm{diff}}=\frac{c}{\tau_{\perp}}, \quad \tau_{\perp}=r_{0} n_{\mathrm{e}} \sigma_{\mathrm{T}}$,

where $n_{\mathrm{e}}$ is the electron number density, $v_{\perp}^{\text {diff }}$ is the photon diffusion velocity perpendicular to the column axis, and $\tau_{\perp}$ is the perpendicular optical thickness. The Thomson cross section $\sigma_{\mathrm{T}}$ is appropriate for photons propagating perpendicular to the column axis (Wang \& Frank 1981). The electron number density $n_{\mathrm{e}}$ appearing in Eq. (11) can be eliminated using the mass conservation relation,

$\dot{M}=\pi r_{0}^{2} n_{\mathrm{e}} m_{\mathrm{p}} v$,

where $\dot{M}$ is the accretion rate and $v$ is the inflow velocity, defined to be positive. Combining relations, we can express the escape time through the walls as

$t_{\mathrm{esc}}=\frac{\dot{M} \sigma_{\mathrm{T}}}{\pi m_{\mathrm{p}} v c}$.

The deceleration in the sinking region begins on the downstream side of the shock, and therefore we set $v=v_{\mathrm{ps}}$ in Eq. (13) and equate $t_{\mathrm{dyn}}$ and $t_{\mathrm{esc}}$ to obtain

$H=\frac{\dot{M} \sigma_{\mathrm{T}}}{2 \pi m_{\mathrm{p}} c}$,

which is essentially the same result obtained by Burnard et al. (1991). Expressing the accretion rate in terms of the luminosity using the relation

$L_{\mathrm{X}}=\frac{G M_{*} \dot{M}}{R_{*}}$

yields the equivalent expression

$H=1.14 \times 10^{5} \mathrm{~cm}\left(\frac{M_{*}}{1.4 M_{\odot}}\right)^{-1}\left(\frac{R_{*}}{10 \mathrm{~km}}\right)\left(\frac{L_{\mathrm{X}}}{10^{37} \mathrm{erg} \mathrm{s}^{-1}}\right)$.

This confirms that the shock is located a few kilometers above the stellar surface in the luminous sources with $L_{X} \sim$ $10^{37-38} \mathrm{erg} \mathrm{s}^{-1}$ (Basko \& Sunyaev 1976). It follows that $R_{*} /(49 H) \ll 1$ for sources close to or above the critical luminosity, and therefore Eq. (9) reduces to

$L_{\text {crit }}=\frac{G M_{*} m_{\mathrm{p}} c}{\sigma_{\|}} \frac{\pi r_{0}^{2}}{R_{*}^{2}}$,

in agreement with Burnard et al. (1991). Note that in this limit, the critical luminosity simply reduces to the effective Eddington value given by Eq. (2).

\subsection{Connection between column radius and Alfvén radius}

In this section, we wish to relate the critical luminosity $L_{\text {crit }}$ in Eq. (17) to the magnetic field strength at the stellar surface, $B_{*}$, by utilizing the connection between the radius of the accretion column, $r_{0}$, and the Alfvén radius in the disk, $R_{\mathrm{A}}$. The inclination angle between the axis of the accretion disk and the star's magnetic axis varies with a period equal to the pulsar's spin period, and this causes an associated variation of the Alfvén radius. However, for our purposes here, an adequate approximation is obtained by using Eq. (13) from Lamb et al. (1973), which yields

$$
\begin{aligned}
R_{\mathrm{A}}= & 2.73 \times 10^{7} \mathrm{~cm}\left(\frac{\Lambda}{0.1}\right)\left(\frac{M_{*}}{1.4 M_{\odot}}\right)^{1 / 7}\left(\frac{R_{*}}{10 \mathrm{~km}}\right)^{10 / 7} \\
& \times\left(\frac{B_{*}}{10^{12} \mathrm{G}}\right)^{4 / 7}\left(\frac{L_{\mathrm{X}}}{10^{37} \mathrm{erg} \mathrm{s}^{-1}}\right)^{-2 / 7},
\end{aligned}
$$

where the constant $\Lambda=1$ for spherical accretion and $\Lambda<1$ for disk accretion. A variety of uncertainties are folded into $\Lambda$, 
such as the spin-averaging of $R_{\mathrm{A}}$ and the possible role of plasma shielding and other magnetospheric effects. Based on Eq. (2) from Harding et al. (1984), $\Lambda$ can be approximated in the disk application using

$\Lambda \approx 0.22 \alpha^{18 / 69}$

where $\alpha<1$ denotes the Shakura-Sunyaev parameter (Shakura $\&$ Sunyaev 1973). Although it is difficult to estimate $\alpha$ with any certainty, we generally expect to find $\alpha \sim 0.01-0.1$. We therefore set $\Lambda=0.1$ in our numerical applications.

The Alfvén radius in the disk is connected with the outer surface of the accretion column through the dipole shape of the pulsar magnetosphere. The equation for the shape of the critical field line as a function of the polar angle $\theta$ is given by the standard dipole formula

$R=R_{\mathrm{A}} \sin ^{2} \theta$.

Setting the radius $R$ equal to the stellar radius $R_{*}$ yields for the critical angle at the outer edge of the accretion column

$\sin ^{2} \theta_{\mathrm{c}}=\frac{R_{*}}{R_{\mathrm{A}}}$

Using the small-angle relation $\theta_{\mathrm{c}} \approx \sin \theta_{\mathrm{c}}$, we obtain for the column radius

$r_{0}=R_{*} \theta_{\mathrm{c}}=R_{*}\left(\frac{R_{*}}{R_{\mathrm{A}}}\right)^{1 / 2}$.

By substituting for the Alfvén radius in Eq. (22) using Eq. (18), we find that the expression for the column radius $r_{0}$ can be rewritten in cgs units as

$$
\begin{aligned}
r_{0}= & 1.93 \times 10^{5} \mathrm{~cm}\left(\frac{\Lambda}{0.1}\right)^{-1 / 2}\left(\frac{M_{*}}{1.4 M_{\odot}}\right)^{-1 / 14}\left(\frac{R_{*}}{10 \mathrm{~km}}\right)^{11 / 14} \\
& \times\left(\frac{B_{*}}{10^{12} \mathrm{G}}\right)^{-2 / 7}\left(\frac{L_{\mathrm{X}}}{10^{37} \mathrm{erg} \mathrm{s}^{-1}}\right)^{1 / 7} \cdot
\end{aligned}
$$

Using Eq. (23) to substitute for $r_{0}$ in Eq. (17), and setting $L_{\mathrm{X}}=$ $L_{\text {crit }}$, we obtain for the critical luminosity the new expression

$$
\begin{aligned}
L_{\text {crit }}= & 7.79 \times 10^{35} \operatorname{erg~s}^{-1}\left(\frac{\Lambda}{0.1}\right)^{-7 / 5}\left(\frac{\sigma_{\|}}{\sigma_{\mathrm{T}}}\right)^{-7 / 5} \\
& \times\left(\frac{M_{*}}{1.4 M_{\odot}}\right)^{6 / 5}\left(\frac{R_{*}}{10 \mathrm{~km}}\right)^{-3 / 5}\left(\frac{B_{*}}{10^{12} \mathrm{G}}\right)^{-4 / 5}
\end{aligned}
$$

where we have also introduced the Thomson cross section $\sigma_{\mathrm{T}}$ as a convenient scaling for the parallel scattering cross section $\sigma_{\|}$. The next step is to evaluate the cross section ratio $\sigma_{\|} / \sigma_{\mathrm{T}}$ in terms of observable source parameters.

\subsection{Electron scattering cross section for parallel propagation}

In typical X-ray pulsars, most of the observed radiation is emitted at energies below the cyclotron energy, $E_{\text {cyc }}$. Hence the crosssection ratio $\sigma_{\|} / \sigma_{\mathrm{T}}$ can be roughly approximated using (e.g., Arons et al. 1987).

$$
\frac{\sigma_{\|}}{\sigma_{\mathrm{T}}}=\left(\frac{\bar{E}}{E_{\mathrm{cyc}}}\right)^{2},
$$

where $\bar{E}$ is a measure of the mean energy of the photons propagating parallel to the magnetic field, and the cyclotron energy $E_{\text {cyc }}$ is given by

$E_{\mathrm{cyc}}=11.58 \mathrm{keV}\left(\frac{B}{10^{12} \mathrm{G}}\right)$.

The mean photon energy $\bar{E}$ in Eq. (25) can be estimated observationally by integrating the spectrum for a given source. However, in luminous X-ray pulsars, most of the observed radiation escapes through the walls of the accretion column, perpendicular to the magnetic field, and therefore the observed spectrum may not be representative of the distribution of photons propagating along the column axis. As an alternative, we can estimate $\bar{E}$ based on the thermal structure of the accreting gas. Specifically, we assume that

$\bar{E}=w k T_{\text {eff }}$,

where $T_{\text {eff }}$ is the effective temperature of the radiation in the post-shock region, $k$ is Boltzmann's constant, and the constant $w$ depends on the shape of the spectrum inside the column. We expect that $w \sim 1-3$, with the lower value corresponding to bremsstrahlung and the upper value to a Planck spectrum. Detailed models suggest that the spectrum inside the column is dominated by bremsstrahlung emission (Becker \& Wolff 2007), and therefore we will set $w=1$ in the numerical results presented later.

The effective temperature is related to the post-shock radiation pressure, $P_{\mathrm{r}}$, via

$a T_{\mathrm{eff}}^{4}=3 P_{\mathrm{r}}$.

The value of $P_{\mathrm{r}}$ can be estimated using the momentum balance relation

$P_{\mathrm{r}}=\rho_{\mathrm{ff}} v_{\mathrm{ff}}^{2}=\frac{\dot{M} v_{\mathrm{ff}}}{\pi r_{0}^{2}}$,

where $\rho_{\mathrm{ff}}$ and $v_{\mathrm{ff}}$ (Eq. (3)) denote the upstream mass density and velocity, respectively, just above the shock. Eliminating $P_{\mathrm{r}}$ between Eqs. (28) and (29), and substituting for $\dot{M}$ and $r_{0}$ using Eqs. (15) and (23), we find that

$$
\begin{aligned}
T_{\text {eff }}= & 4.35 \times 10^{7} \mathrm{~K}\left(\frac{\Lambda}{0.1}\right)^{1 / 4}\left(\frac{M_{*}}{1.4 M_{\odot}}\right)^{-5 / 56}\left(\frac{R_{*}}{10 \mathrm{~km}}\right)^{-15 / 56} \\
& \times\left(\frac{B_{*}}{10^{12} \mathrm{G}}\right)^{1 / 7}\left(\frac{L_{\mathrm{X}}}{10^{37} \mathrm{erg} \mathrm{s}^{-1}}\right)^{5 / 28} .
\end{aligned}
$$

Combining Eqs. (25)-(27), and (30), we obtain for the required cross section ratio the result

$$
\begin{aligned}
\frac{\sigma_{\|}}{\sigma_{\mathrm{T}}}= & 0.106\left(\frac{\Lambda}{0.1}\right)^{1 / 2} w^{2}\left(\frac{M_{*}}{1.4 M_{\odot}}\right)^{-5 / 28}\left(\frac{R_{*}}{10 \mathrm{~km}}\right)^{-15 / 28} \\
& \times\left(\frac{B_{*}}{10^{12} \mathrm{G}}\right)^{-12 / 7}\left(\frac{L_{\mathrm{X}}}{10^{37} \mathrm{erg} \mathrm{s}^{-1}}\right)^{5 / 14} .
\end{aligned}
$$

Using this result to substitute for $\sigma_{\|} / \sigma_{\mathrm{T}}$ in Eq. (24) yields the final expression for the critical luminosity as a function of the surface magnetic field strength,

$$
\begin{aligned}
L_{\text {crit }}= & 1.49 \times 10^{37} \operatorname{erg~s}^{-1}\left(\frac{\Lambda}{0.1}\right)^{-7 / 5} w^{-28 / 15} \\
& \times\left(\frac{M_{*}}{1.4 M_{\odot}}\right)^{29 / 30}\left(\frac{R_{*}}{10 \mathrm{~km}}\right)^{1 / 10}\left(\frac{B_{*}}{10^{12} \mathrm{G}}\right)^{16 / 15} .
\end{aligned}
$$


For typical neutron star parameters, with $M_{*}=1.4 M_{\odot}$, $R_{*}=10 \mathrm{~km}, \Lambda=0.1$, and $w=1$, we obtain $L_{\text {crit }}=$ $1.49 \times 10^{37} \mathrm{erg} \mathrm{s}^{-1} B_{12}^{16 / 15}$, where $B_{12}$ is the surface magnetic field strength in units of $10^{12} \mathrm{G}$. In Sect. 4 we plot the critical luminosity and compare it with the variability data for several XRBPs.

\section{Variation of emission height}

The new expression for the critical luminosity given by Eq. (32) allows us to separate accretion-powered X-ray pulsars into subcritical and supercritical categories. Our hypothesis is that in the subcritical sources with variable luminosity $L_{X}$, the cyclotron energy $E_{\text {cyc }}$ will exhibit a positive correlation with $L_{X}$, and in the supercritical sources the reverse behavior will be observed. The observed CRSF is imprinted on the spectrum at the altitude where most of the emitted radiation escapes from the accretion column. In order to quantify the expected behaviors in the subcritical and supercritical regimes, we must therefore explore the expected variation of the emission height as a function of luminosity for both types of sources. The geometry of the super- and subcritical sources is depicted schematically in Fig. 1.

\subsection{Supercritical sources}

In the supercritical sources (luminosity $L_{X} \gtrsim L_{\text {crit }}$ ), radiation pressure dominates the flow dynamics all the way to the stellar surface. Inside the radiation-dominated shock, the infalling matter begins to decelerate as it first encounters the "cushion" of radiation hovering at the shock altitude (Davidson 1973). At this altitude, there is a local balance between downward advection and upward diffusion of radiation, and therefore the photon distribution is roughly static. Most of the kinetic energy of the accretion flow is carried away by the scattered radiation, which is likely to be beamed in the downward direction due to specialrelativistic aberration (e.g., Ferrigno et al. 2009). Below the shock altitude, the photons are trapped by advection, although they eventually manage to escape by diffusing through the walls of the column. Hence the observed radiation does not escape from the shock altitude $H$, but rather from a lower altitude.

Our goal here is to estimate the typical altitude, denoted by $h_{\mathrm{s}}$, at which the photons diffuse through the column walls to form the observed X-ray spectrum in the supercritical case. We assume that the observed CRSF is imprinted at this altitude, because at higher altitudes the photons have not had enough time to diffuse through the column and escape through the walls. Conversely, at lower altitudes, the increasing density of the gas in the column inhibits the escape of radiation. We therefore expect that the CRSF energy will reflect the cyclotron energy at the altitude $z=h_{\mathrm{s}}$ where the final deceleration phase begins.

We can estimate the emission height $h_{\mathrm{s}}$ in the supercritical sources by ensuring that all of the kinetic energy is radiated through the walls by the escaping photons in the altitude range $0<z<h_{\mathrm{s}}$ (Basko \& Sunyaev 1976). Working in the frame comoving with the mean vertical velocity of the radiation in the accretion column, we note that the fraction of the radiation escaping through the walls in the comoving time interval $\mathrm{d} t^{\prime}$ is equal to $\mathrm{d} t^{\prime} / t_{\text {esc }}$, where $t_{\text {esc }}$ is the escape time given by Eq. (13). The requirement that all of the radiation escapes by the time the matter reaches the stellar surface is therefore expressed by the integral condition

$\int_{0}^{h_{\mathrm{s}}}\left|\frac{\mathrm{d} t^{\prime}}{\mathrm{d} z^{\prime}}\right| \frac{\mathrm{d} z^{\prime}}{t_{\mathrm{esc}}\left(z^{\prime}\right)}=1$.
Using Eq. (13) to substitute for $t_{\mathrm{esc}}$ yields

$\int_{0}^{h_{\mathrm{s}}} \frac{\pi m_{\mathrm{p}} v c}{\dot{M} \sigma_{\mathrm{T}}} \frac{\mathrm{d} z^{\prime}}{v_{\text {eff }}}=1$,

where the effective velocity for the photon transport is defined by

$v_{\text {eff }} \equiv\left|\frac{\mathrm{d} z^{\prime}}{\mathrm{d} t^{\prime}}\right|$.

The flow is expected to be almost perfectly "trapped" in the region below $z^{\prime}=h_{\mathrm{s}}$, meaning that advection and diffusion are nearly balanced, leaving very little net flux of radiation (Becker 1998). This implies that the effective velocity $v_{\text {eff }}$ is much smaller than the flow velocity $v$. We define the parameter $\xi$ as the ratio of these two velocities,

$\xi \equiv \frac{v_{\mathrm{eff}}}{v}$

We demonstrate in Appendix A that the value of $\xi$ can be estimated using

$\xi=\frac{1}{\mathcal{M}_{\infty}^{2}}$,

where $\mathcal{M}_{\infty}$ denotes the incident (upstream) Mach number of the flow with respect to the radiation sound speed. In the sinking region below the shock, the effective velocity approaches zero as the gas settles onto the stellar surface. The relationship between the upstream Mach number $\mathcal{M}_{\infty}$ and the X-ray luminosity $L_{X}$ is plotted in Fig. 12 from Becker (1998). For the parameter range of interest here, it is sufficient to adopt a constant value for $\xi$ in the range $\xi \sim 10^{-2}-10^{-3}$. The low value for the effective velocity tends to make the emission region more compact in the supercritical sources.

Combining relations, we find that

$\int_{0}^{h_{\mathrm{s}}} \frac{\pi m_{\mathrm{p}} c}{\dot{M} \sigma_{\mathrm{T}} \xi} \mathrm{d} z^{\prime}=1$,

and therefore the altitude of the emission region is given by

$h_{\mathrm{s}}=\frac{\dot{M} \sigma_{\mathrm{T}} \xi}{\pi m_{\mathrm{p}} c}=\frac{L_{\mathrm{X}} R_{*} \sigma_{\mathrm{T}} \xi}{\pi m_{\mathrm{p}} c G M_{*}}$

where the final result follows from Eq. (15). We can also express $h_{\mathrm{s}}$ in cgs units using

$$
\begin{aligned}
h_{\mathrm{S}}= & 2.28 \times 10^{3} \mathrm{~cm}\left(\frac{\xi}{0.01}\right)\left(\frac{M_{*}}{1.4 M_{\odot}}\right)^{-1} \\
& \times\left(\frac{R_{*}}{10 \mathrm{~km}}\right)\left(\frac{L_{\mathrm{X}}}{10^{37} \mathrm{erg} \mathrm{s}^{-1}}\right) .
\end{aligned}
$$

Note that the emission height in the supercritical sources varies in proportion to the luminosity $L_{X}$ (see Fig. 1).

Based on Eqs. (16) and (40), we conclude that

$\frac{h_{\mathrm{s}}}{H}=2.0 \xi \ll 1$,

and therefore the characteristic height of emission in the supercritical sources is located far below the altitude of the radiationdominated shock. Eq. (40) indicates that the height of the emission region $h_{\mathrm{s}}$ scales in proportion to the luminosity $L_{\mathrm{X}}$ in the supercritical sources, which is consistent with the observed behavior in the group 1 sources (Klochkov et al. 2011). 


\subsection{Subcritical sources}

In the subcritical sources (luminosity $L_{X} \lesssim L_{\text {crit }}$ ), the matter still passes through a radiation-dominated shock, which accomplishes the initial deceleration, but the pressure of the radiation is insufficient to bring the matter to rest at the stellar surface (Basko \& Sunyaev 1976). In this case, the final stopping occurs via direct Coulomb interactions close to the base of the accretion column (Burnard et al. 1991). Our goal in this section is to estimate the characteristic emission height for the subcritical sources, denoted by $h_{\mathrm{c}}$, which is the altitude at which Coulomb interactions begin to decelerate the plasma to rest. The emission is expected to be concentrated in this region because this is essentially the first opportunity that the radiation inside the column has to diffuse through the walls. At higher altitudes, the radiation is swept along by advection, and there is not enough time for the photons to escape. At lower altitudes, the radiation is trapped in the column due to the increasing density, and therefore the emission through the walls tapers off as $z \rightarrow 0$. Hence we expect that in the subcritical case, the CRSF energy is imprinted at the altitude $z=h_{\mathrm{c}}$ where the strong Coulomb deceleration begins.

The Thomson optical depth, $\tau_{*}$, required to stop the flow via Coulomb interactions can be estimated in the typical pulsar magnetic field regime using Eq. (3.34) from Nelson et al. (1993) to write

$\tau_{*}=51.4\left(\frac{M_{*}}{1.4 M_{\odot}}\right)^{2}\left(\frac{R_{*}}{10 \mathrm{~km}}\right)^{-2} \frac{1}{\ln \left(2 n_{\max }\right)}$,

where the maximum excited Landau level, $n_{\max }$, is given by

$n_{\max }=\frac{m_{\mathrm{e}} v_{\mathrm{ff}}^{2}}{2 E_{\mathrm{cyc}}}$.

A summary of the derivation leading up to Eq. (42) is provided in Appendix B. Adopting typical X-ray pulsar parameters, we find that $\tau_{*} \sim 20$, which is the value utilized in our numerical examples.

We can use Eq. (42) to estimate the emission height in the subcritical sources, $h_{\mathrm{c}}$, as follows. The Thomson depth $\tau$ as a function of the altitude $z$ measured from the stellar surface is computed using

$\tau(z)=\int_{0}^{z} \frac{\rho\left(z^{\prime}\right) \sigma_{\mathrm{T}}}{m_{\mathrm{p}}} \mathrm{d} z^{\prime}$

where $\rho=n_{\mathrm{e}} m_{\mathrm{p}}$ is the mass density, given by (see Eq. (12))

$\rho(z)=\frac{\dot{M}}{\pi r_{0}^{2} v(z)}$.

Assuming that the gas decelerates uniformly in the Coulomb stopping region (starting at altitude $h_{\mathrm{c}}$ ) from the post-shock velocity $v_{\mathrm{ps}}$, we find that the required deceleration is given by $a=v_{\mathrm{ps}}^{2} /\left(2 h_{\mathrm{c}}\right)$ (cf. Eq. (6)). The velocity profile $v(z)$ associated with the constant deceleration $a$ is computed using the standard kinematical relations

$a=-\frac{\mathrm{d} v}{\mathrm{~d} t}=v \frac{\mathrm{d} v}{\mathrm{~d} z}=\frac{1}{2} \frac{\mathrm{d} v^{2}}{\mathrm{~d} z}$,

where the negative sign appears because we have defined $v$ and $a$ to be positive. Setting $v_{\mathrm{ps}}=v_{\mathrm{ff}} / 7$ (see Eq. (4)), we obtain for the deceleration $a=v_{\mathrm{ff}}^{2} /\left(98 h_{\mathrm{c}}\right)$. Upon integration of Eq. (46), we therefore find that the velocity profile in the Coulomb stopping region is given by

$v(z)=\frac{v_{\mathrm{ff}}}{7} \sqrt{\frac{z}{h_{\mathrm{c}}}}$,

where $v_{\mathrm{ff}}$ is evaluated using Eq. (3).
Using Eq. (47) to substitute for $v(z)$ in Eq. (45) and carrying out the integration in Eq. (44), we obtain for the optical depth profile

$\tau(z)=\frac{14 \dot{M} \sigma_{\mathrm{T}}}{\pi r_{0}^{2} m_{\mathrm{p}}}\left(\frac{2 G M_{*}}{R_{*}}\right)^{-1 / 2} \sqrt{h_{\mathrm{c}} z}$.

Finally, setting $z=h_{\mathrm{c}}$ and $\tau=\tau_{*}$, we find that the Thomson optical depth required for Coulomb stopping is given by

$\tau_{*}=\frac{14 \dot{M} \sigma_{\mathrm{T}} h_{\mathrm{c}}}{\pi r_{0}^{2} m_{\mathrm{p}}}\left(\frac{2 G M_{*}}{R_{*}}\right)^{-1 / 2}$,

which can be rearranged to obtain for the subcritical emission height

$h_{\mathrm{c}}=\frac{\pi r_{0}^{2} m_{\mathrm{p}} \tau_{*}}{14 \dot{M} \sigma_{\mathrm{T}}}\left(\frac{2 G M_{*}}{R_{*}}\right)^{1 / 2}$.

Substituting for $\dot{M}$ and $r_{0}$ using Eqs. (15) and (23), respectively, yields the equivalent cgs expression

$$
\begin{aligned}
h_{\mathrm{c}}= & 1.48 \times 10^{5} \mathrm{~cm}\left(\frac{\Lambda}{0.1}\right)^{-1}\left(\frac{\tau_{*}}{20}\right)\left(\frac{M_{*}}{1.4 M_{\odot}}\right)^{19 / 14}\left(\frac{R_{*}}{10 \mathrm{~km}}\right)^{1 / 14} \\
& \times\left(\frac{B_{*}}{10^{12} \mathrm{G}}\right)^{-4 / 7}\left(\frac{L_{\mathrm{X}}}{10^{37} \mathrm{erg} \mathrm{s}^{-1}}\right)^{-5 / 7} .
\end{aligned}
$$

This result indicates that the emission height in the subcritical sources decreases with increasing luminosity, which is consistent with the behavior observed in the group 2 sources (Staubert et al. 2007). As indicated in Fig. 1, in the subcritical case, a decrease in the luminosity causes the beam pattern to transition from a pure fan configuration to a hybrid pattern that includes a pencil component. At very low luminosities, the shock essentially sits on the stellar surface, and the emission occurs via the pencil component only (Burnard et al. 1991; Nelson et al. 1993).

\subsection{Transition from Coulomb stopping to gas shock}

Equation (51) gives the height of the emission zone in the subcritical case, based on the assumption that the final deceleration to rest at the stellar surface occurs via Coulomb interactions. We can estimate the minimum luminosity (or accretion rate) such that Coulomb interactions are capable of stopping the flow by requiring that $h_{\mathrm{c}}<H$, where $H$ is the altitude of the radiationdominated shock. If this condition is violated, then the incident velocity of the gas entering the Coulomb deceleration region becomes essentially the full free-fall velocity, $v_{\mathrm{ff}}$, rather than the reduced post-shock velocity, $v_{\mathrm{ps}}=v_{\mathrm{ff}} / 7$. The associated drop in the density causes the Thomson depth $\tau$ to fall below the value $\tau_{*} \sim 20$ required for the gas to be effectively stopped via Coulomb interactions. It is not completely clear what happens in this case, but we expect that the final phase of deceleration will occur via passage through a gas-mediated shock near the stellar surface (Langer \& Rappaport 1982).

By combining Eqs. (14), (15) and (50), we can show that the condition $h_{\mathrm{c}}<H$ implies that

$L_{\mathrm{X}}>\frac{2^{1 / 4} \pi m_{\mathrm{p}} r_{0}}{\sigma_{\mathrm{T}}}\left(\frac{\tau_{*} c}{7}\right)^{1 / 2}\left(\frac{G M_{*}}{R_{*}}\right)^{5 / 4}$.

Substituting for $r_{0}$ using Eq. (23) and rearranging, we obtain

$L_{\mathrm{X}}>L_{\text {coul }}$, 

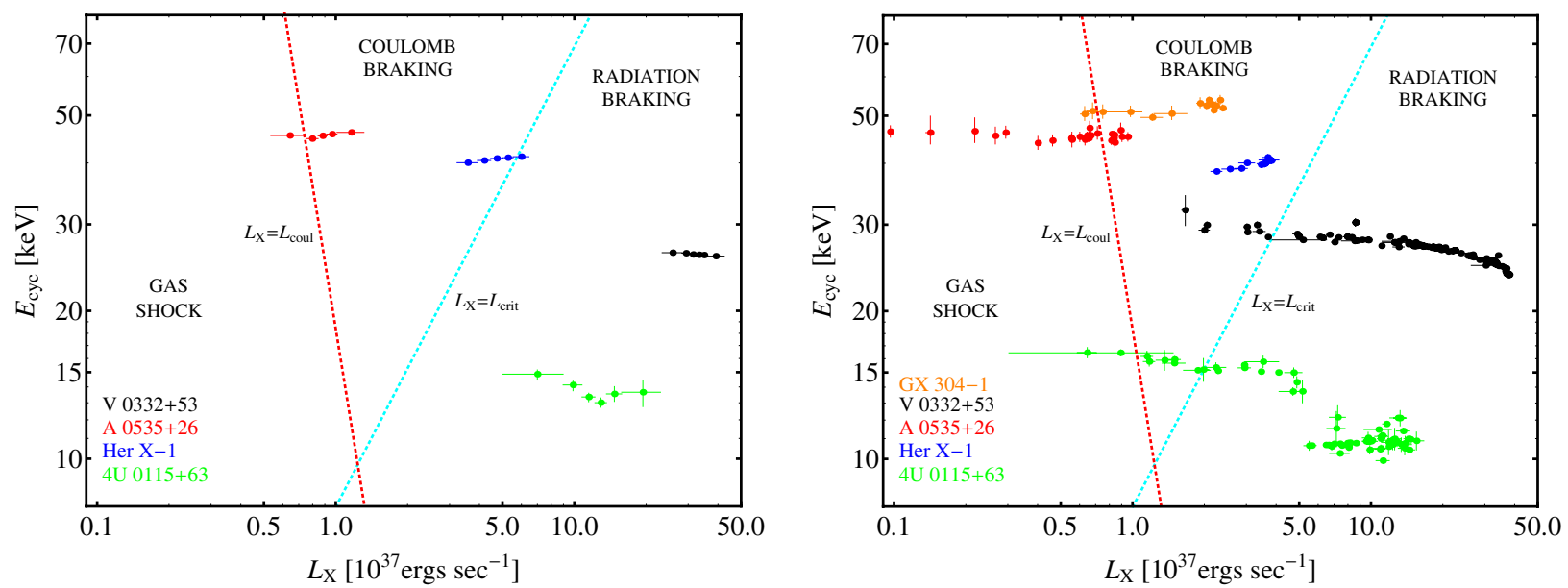

Fig. 2. Variability of the cyclotron line energy with luminosity for different sources. The blue dashed line represents the critical luminosity, plotted by setting $L_{\mathrm{X}}=L_{\text {crit }}$ and $E_{\text {cyc }}=E_{*}$, where $L_{\text {crit }}$ is evaluated using Eq. (55). The red dashed line represents the Coulomb stopping luminosity, plotted by setting $L_{\mathrm{X}}=L_{\text {coul }}$ and $E_{\text {cyc }}=E_{*}$, where $L_{\text {coul }}$ is evaluated using Eq. (59). Left: observations on a pulse-to-pulse timescale. Right: observations on longer timescales.

Table 1. Source sample characteristics.

\begin{tabular}{lccccc}
\hline \hline Source & Instrument & $\begin{array}{c}\text { Long-term } \\
\text { correlation }\end{array}$ & $\begin{array}{c}\text { Pulse-pulse } \\
\text { correlation }^{-}\end{array}$ & $\begin{array}{c}\Delta E \\
(\mathrm{keV})\end{array}$ & $\begin{array}{c}\text { Distance } \\
(\mathrm{kpc})\end{array}$ \\
\hline 4U 0115+63 & INTEGRAL/RXTE & $\begin{array}{c}\text { cont. dependent }^{1} \\
\text { negative }^{2}\end{array}$ & negative $^{6}$ & {$[3-60]$} & $8.0^{7}$ \\
V0332+53 & INTEGRAtive & {$[3-100]$} & $7.5^{8}$ \\
Her X-1 & RXTE & positive $^{3}$ & positive $^{6}$ & {$[5-60]$} & $6.4^{9}$ \\
A0535+26 & INTEGRAL/RXTE & no $^{4}$ & positive $^{6}$ & {$[3-50]$} & $2.0^{10}$ \\
GX 304-1 & RXTE/Suzaku & positive $^{5}$ & - & {$[3-100]$} & $2.4^{11}$ \\
\hline
\end{tabular}

References. ${ }^{(1)}$ Müller et al. (2011); (2) Tsygankov et al. (2010); (3) Staubert et al. (2007); ${ }^{(4)}$ Caballero et al. (2007); (5) Yamamoto et al. (2011); (6) Klochkov et al. (2011); (7) Negueruela \& Okazaki (2001); ${ }^{(8)}$ Negueruela et al. (1999); ${ }^{(9)}$ Reynolds et al. (1997); (10) Steele et al. (1998); (11) Parkes et al. (1980).

Notes. Our analysis is based on a combination of published data (references given) and reprocessed observational data for a number of cyclotron line sources (Col. 1) as observed by different X-ray observatories (Col. 2), listed here. The cyclotron line sources have been observed to show different types of correlation, or no correlation, of their cyclotron line energies with changes in the X-ray luminosity.

where

$$
\begin{aligned}
L_{\mathrm{coul}}= & 1.17 \times 10^{37} \mathrm{erg} \mathrm{s}^{-1}\left(\frac{\Lambda}{0.1}\right)^{-7 / 12}\left(\frac{\tau_{*}}{20}\right)^{7 / 12}\left(\frac{M_{*}}{1.4 M_{\odot}}\right)^{11 / 8} \\
& \times\left(\frac{R_{*}}{10 \mathrm{~km}}\right)^{-13 / 24}\left(\frac{B_{*}}{10^{12} \mathrm{G}}\right)^{-1 / 3} \cdot
\end{aligned}
$$

For luminosities $L_{\mathrm{X}} \lesssim L_{\text {coul }}$, we expect that the characteristic emission height settles down onto the stellar surface. For very low luminosities, $L_{\mathrm{X}} \lesssim 10^{34-35} \mathrm{erg} \mathrm{s}^{-1}$, the radiationdominated shock and the Coulomb atmosphere both dissipate, and the matter strikes the stellar surface after passing through a gas-mediated shock (Langer \& Rappaport 1982), as indicated in Fig. 1.

\section{Applications}

Our final result for the critical luminosity as a function of the surface magnetic field strength $B_{*}$ is given by Eq. (32), which can be rewritten as

$$
\begin{aligned}
L_{\text {crit }}= & 1.28 \times 10^{37} \mathrm{erg} \mathrm{s}^{-1}\left(\frac{\Lambda}{0.1}\right)^{-7 / 5} w^{-28 / 15} \\
& \times\left(\frac{M_{*}}{1.4 M_{\odot}}\right)^{29 / 30}\left(\frac{R_{*}}{10 \mathrm{~km}}\right)^{1 / 10}\left(\frac{E_{*}}{10 \mathrm{keV}}\right)^{16 / 15},
\end{aligned}
$$

where

$$
E_{*}=11.58 \mathrm{keV}\left(\frac{B_{*}}{10^{12} \mathrm{G}}\right)
$$

denotes the surface value of the CRSF energy. This relation is indicated by the dashed blue line in Fig. 2. Sources to the right of this line are radiating supercritically, and consequently radiation pressure accomplishes the deceleration all the way down to the stellar surface. For sources to the left of this line, the final deceleration occurs via Coulomb interactions.

It is now interesting to compute the critical luminosity for a number of sources and to compare it with the observed variability of $E_{\text {cyc }}$ as a function of $L_{\mathrm{X}}$ for subcritical and supercritical cases. We have selected for this study the sources for which the behavior of the cyclotron line energy with luminosity has been studied sufficiently well, including both the variations on long timescales (days to months) and short timescales ("pulseto-pulse"). Our source sample is listed in Table 1, where we also summarize the reported $E_{\text {cyc }}-L_{X}$ behavior (positive/negative correlation or no dependence) for each source. The two panels in Fig. 2 depict the variability of $E_{\text {cyc }}$ with luminosity both on "pulse-to-pulse" (left) and longer (right) timescales for all of the sources considered here. The corresponding references for the data are given in Table 1. The data for the longer-term variability are based on studies of the phase-averaged emission conducted either during outbursts of the transient sources 

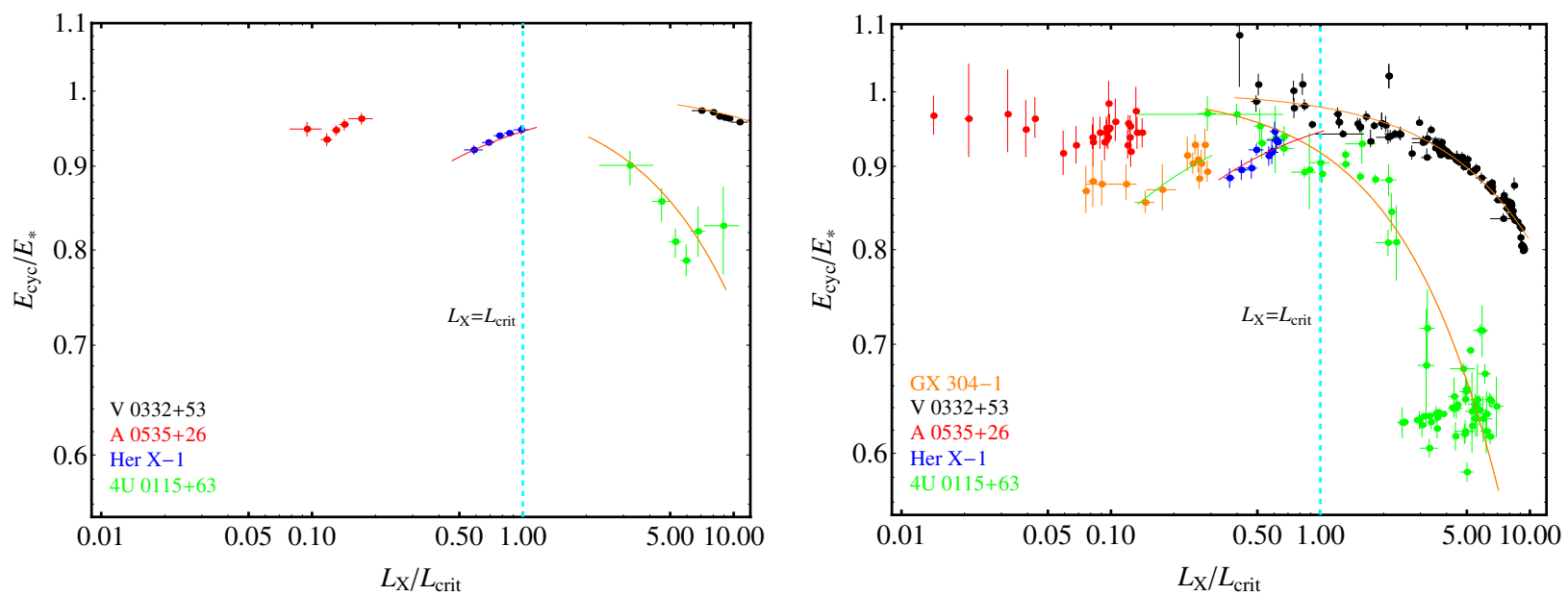

Fig. 3. Same as Fig. 2 except with the $x$-axis rescaled as $L_{\mathrm{X}} / L_{\text {crit }}$ and the $y$-axis rescaled as $E_{\text {cyc }} / E_{*}$. The values of $E_{*}$ and $L_{\text {crit }}$ used for each source are listed in Table 2. The solid curves associated with each group of data represent the theoretical predictions for the variation of the CRSF energy $E_{\text {cyc }}$ as a function of $L_{\mathrm{X}}$ for each source, computed using Eq. (58), with the emission height $h$ evaluated using Eq. (40) for the supercritical sources and Eq. (51) for the subcritical sources.

Table 2. Theoretical parameters for each source, based on analysis of the pulse-to-pulse variability and the longer-term variability.

\begin{tabular}{lcccccc}
\hline \hline Source & $\begin{array}{c}\text { Long-term } \\
\xi\end{array}$ & $\begin{array}{c}\text { Long-term } E_{*} \\
{[\mathrm{keV}]}\end{array}$ & $\begin{array}{c}\text { Long-term } L_{\text {crit }} \\
{\left[10^{37} \mathrm{erg} \mathrm{s}^{-1}\right]}\end{array}$ & $\begin{array}{c}\text { Pulse-pulse } \\
\xi\end{array}$ & $\begin{array}{c}\text { Pulse-pulse } E_{*} \\
{[\mathrm{keV}]}\end{array}$ & $\begin{array}{c}\text { Pulse-pulse } L_{\text {crit }} \\
{\left[10^{37} \mathrm{erg} \mathrm{s}^{-1}\right]}\end{array}$ \\
\hline 4U 0115+63 & $5.72 \times 10^{-2}$ & 17.0 & 2.24 & $2.14 \times 10^{-2}$ & 16.5 & 2.17 \\
V 0332+53 & $7.86 \times 10^{-3}$ & 29.7 & 4.06 & $1.43 \times 10^{-3}$ & 27.0 & 3.67 \\
Her X-1 & - & 43.5 & 6.11 & - & 43.5 & 6.11 \\
A 0535+26 & - & 48.0 & 6.78 & - & 48.0 & 6.78 \\
GX 304-1 & - & 58.0 & 8.30 & - & - & - \\
\hline
\end{tabular}

Notes. The parameter $\xi$ is relevant only for the two supercritical sources, 4U 0115+63 and V 0332+53 (see Sect. 3.1).

(Tsygankov et al. 2007, 2010), or following the long-term variation of the emission from the persistent sources (Staubert et al. 2007). For the pulse-to-pulse variability we refer to the results of Klochkov et al. (2011) who have shown that for a set of pulsars the cyclotron line energy varies with the amplitude of individual pulses. This amplitude most probably reflects the instantaneous mass accretion rate.

The luminosities for A 0535+26, 4 U0115+63 and Her X-1 were calculated by integrating the flux of each source over a nearly identical energy range $\Delta E$ (see Table 1$)$. For V 0332+53 (Tsygankov et al. 2010) and GX 304-1 (Yamamoto et al. 2011) published values for $L_{\mathrm{X}}$ and $E_{\mathrm{cyc}}$ were taken, where the luminosity has been calculated over a slightly larger energy range [3-100] keV. The luminosities were computed using the source distances listed in Table 1. It should be noted that uncertainties in the distances will create additional uncertainties in our computed luminosities, which have not been considered here. However, the typical effective uncertainties of $\sim 10 \%$ in the calculated luminosities do not strongly affect our results.

One can see that the sign of the correlation between the cyclotron line energy and the luminosity, when detected, is consistent between the long-term and pulse-to-pulse studies. This suggests that both are reflecting the same underlying physics. It is clear that the variation of $E_{\mathrm{cyc}}$ with $L_{\mathrm{X}}$ for sources on each side of the critical line $L_{X}=L_{\text {crit }}$ is qualitatively consistent with the theoretical predictions, i.e., the correlation between $E_{\text {cyc }}$ and $L_{X}$ is negative for the supercritical sources, and positive for the subcritical ones, reflecting the positive variation of the emission height $h=h_{\mathrm{s}}$ (Eq. (40)) with increasing $L_{\mathrm{X}}$ for the former sources and the negative variation of the emission height $h=h_{\mathrm{c}}$
(Eq. (51)) for the latter sources. In the next section, we explore the variation of $E_{\text {cyc }}$ as a function of $L_{X}$ in more detail.

\subsection{Variation of $E_{c y c}$ with $L_{X}$}

The observed value of $E_{\text {cyc }}$ is connected with the local field strength $B$ at the emission altitude $h$ via Eq. (26), where $B$ has the dipole dependence

$$
\frac{B(R)}{B_{*}}=\left(\frac{R}{R_{*}}\right)^{-3}, \quad R=R_{*}+h .
$$

The corresponding variation of $E_{\mathrm{cyc}}$ as a function of the emission height $h$ is therefore given by

$\frac{E_{\mathrm{cyc}}}{E_{*}}=\left(\frac{R_{*}+h}{R_{*}}\right)^{-3}$,

where $E_{*}$ is the surface value for the cyclotron energy, computed using Eq. (56). Note that we have neglected the variation of the gravitational redshift factor, which is reasonable given the level of approximation employed here (Staubert et al. 2007). In applying Eq. (58) to the supercritical and subcritical cases, we set $h=h_{\mathrm{s}}$ (Eq. (40)) and $h=h_{\mathrm{c}}$ (Eq. (51)), respectively. Hence Eq. (58) can be used to develop theoretical predictions for the variation of $E_{\mathrm{cyc}}$ as a function of $L_{\mathrm{X}}$ for supercritical and subcritical sources.

In Fig. 3 we replot the Fig. 2 data based on a rescaling of the vertical and horizontal axes using the parameters $E_{*}$ and $L_{\text {crit }}$, respectively, which are related to each other via Eq. (55). The value of $L_{\text {crit }}$ used for each source is listed in Table 2, along with 
the corresponding value for the surface cyclotron energy $E_{*}$ obtained by comparing the theoretical variation of $E_{\text {cyc }}$ with the observed variation for each source. In computing $L_{\text {crit }}$, we assume for all sources the same canonical neutron star mass and radius values $M_{*}=1.4 M_{\odot}$ and $R_{*}=10 \mathrm{~km}$, and we set $\Lambda=0.1$ and $w=1$ based on the theoretical considerations discussed above. The vertical dashed line marked $L_{\mathrm{X}}=L_{\text {crit }}$ separates the sources into their sub- and supercritical luminosity states. It should be noted that, as $M_{*}$ and $R_{*}$ are also input parameters for $L_{\text {crit }}$, the exact positioning of the source data on the $x$-axis is driven also by the assumed canonical mass and radius values, which might in fact differ between the individual sources.

Figure 3 also includes curves representing the expected theoretical variation of the CRSF energy $E_{\mathrm{cyc}}$ as a function of the luminosity $L_{\mathrm{X}}$, computed using Eq. (58) with either $h=h_{\mathrm{s}}$ (Eq. (40)) for the supercritical sources or $h=h_{\mathrm{c}}$ (Eq. (51)) for the subcritical ones. We again adopt the canonical values $M_{*}=1.4 M_{\odot}, R_{*}=10 \mathrm{~km}, \Lambda=0.1$, and $w=1$, and we set the Coulomb stopping optical depth using $\tau_{*}=20$. The values of $E_{*}$ and $\xi$ are varied for each source so as to improve the agreement with the data (see Table 2). The parameter $\xi$ is only relevant for the supercritical sources. The values of $\xi$ reported in Table 2 for $\mathrm{V} 0332+53$ and $4 \mathrm{U} 0115+63$ are in the range $\xi \sim 10^{-2}-10^{-3}$, as expected for marginally trapped accretion columns (Becker 1998). Based on the results depicted in Fig. 3, we conclude that the agreement between the observed variation of $E_{\text {cyc }}$ and that predicted by the theoretical models developed here is reasonably close for both the supercritical and subcritical sources.

A special case is $4 \mathrm{U} 0115+63$. This source was previously observed to show an anticorrelation (e.g. Tsygankov et al. 2007 and references therein). The right panels of our Figs. 2 and 3 (observations on longer timescales) include the results for $E_{\text {cyc }}$ and $L_{X}$ obtained by Tsygankov et al. (2007). However, recent studies have shown that the presence of the anticorrelation depends on the choice of the continuum model (Müller et al. 2011).

\subsection{Comparison of $L_{x}$ with $L_{\text {coul }}$}

It is also interesting to compare the observed luminosities with the minimum value, $L_{\text {coul }}$, required for Coulomb stopping to decelerate the flow to rest at the stellar surface, given by Eq. (54). By combining Eqs. (54) and (56), we find that $L_{\text {coul }}$ is related to $E_{*}$ via

$$
\begin{aligned}
L_{\mathrm{coul}}= & 1.23 \times 10^{37} \mathrm{erg} \mathrm{s}^{-1}\left(\frac{\Lambda}{0.1}\right)^{-7 / 12}\left(\frac{\tau_{*}}{20}\right)^{7 / 12}\left(\frac{M_{*}}{1.4 M_{\odot}}\right)^{11 / 8} \\
& \times\left(\frac{R_{*}}{10 \mathrm{~km}}\right)^{-13 / 24}\left(\frac{E_{*}}{10 \mathrm{keV}}\right)^{-1 / 3} .
\end{aligned}
$$

This relation is indicated by the dashed red line in Fig. 2. For sources to the left of this line, we expect that the effect of Coulomb interactions is reduced, and the final stopping occurs via passage through a discontinuous, gas-mediated shock (Langer \& Rappaport 1982). Hence we anticipate that the emission region approaches the stellar surface as $L_{\mathrm{X}}$ is reduced below $L_{\text {coul }}$. This interpretation is consistent with the observational data plotted in Figs. 2 and 3, which indicate that $E_{\text {cyc }}$ approaches a constant value in the limit $L_{\mathrm{X}} / L_{\text {coul }} \ll 1$.

Following our hypothesis, sources in their supercritical state $\left(L_{\mathrm{X}} / L_{\text {crit }} \gtrsim 1\right)$ should display a negative correlation between the luminosity and the cyclotron energy while sources in the subcritical state $\left(L_{X} / L_{\text {crit }} \lesssim 1\right)$ should display the reverse behavior. V 0332+53 and Her X-1 in their super- and subcritical luminosity states nicely fit into that hypothesis on both long and very short timescales. In particular, we note that the model parameters listed in Table 2 for Her X-1 are the same for both the longterm and pulse-to-pulse data. Hence the model developed here for the variation of $E_{\mathrm{cyc}}$ as a function of $L_{\mathrm{X}}$ provides a robust connection with the data across the entire range of luminosity variation for this source, which implies that the underlying physical mechanism of variation is the same for the long-term and pulse-to-pulse variations.

The subcritical source A $0535+26$ shows no significant trend on long timescales (Fig. 2, right), perhaps due to the fact that the luminosity is always close to or below the Coulomb stopping limit, $L_{\text {coul }}$, which suggests that we should expect little variation of $E_{\text {cyc }}$ with $L_{\mathrm{X}}$. On pulse-to-pulse timescales, A $0535+26$ shows some suggestion of a positive correlation (Fig. 2, left), as expected for a subcritical source. The positive correlation suggested by the short-timescale data may reflect the fact that the luminosity is somewhat higher than $L_{\text {coul }}$, which places it in the subcritical regime according to our theory. However, we note that our model for the variation of $E_{\text {cyc }}$ with $L_{X}$ does not work well for A $0535+26$, unless we choose an unreasonably large value for $E_{*}$ relative to the observational data. We believe this reflects the inapplicability of our model in very low-luminosity sources with $L_{\mathrm{X}} \lesssim L_{\text {coul }}$. For GX 304-1, only an indication of a positive $E_{\mathrm{cyc}}-L_{\mathrm{X}}$ correlation, consistent with its subcritical state, can be seen, as also reported by Yamamoto et al. (2011). No pulse-to-pulse spectra are yet available for GX 304-1.

\section{Conclusions}

We have examined the hypothesis that observed bimodal variability of the CRSF energy $E_{\text {cyc }}$ with luminosity $L_{X}$ in accretionpowered X-ray pulsars reflects the dominant mode of accretion, as proposed by Staubert et al. (2007) and Klochkov et al. (2011). In particular, we have derived an expression for the critical luminosity $L_{\text {crit }}$ such that the dynamics in the supercritical sources is determined by the radiation pressure, and the dynamics in the subcritical sources is determined by a combination of radiation pressure and either Coulomb interactions or gas pressure. The detailed formula for $L_{\text {crit }}$ is given by Eq. (32), but essentially we find that for typical neutron star parameters, $L_{\text {crit }} \sim 1.5 \times 10^{37} B_{12}^{16 / 15} \mathrm{erg} \mathrm{s}^{-1}$, where $B_{12}$ is the surface magnetic field strength in units of $10^{12} \mathrm{G}$.

The formula for the critical luminosity was evaluated for 5 sources, based on the maximum value for the CRSF centroid energy for each source, $E_{*}$, which is treated as a variable parameter in our approach. The results obtained for $E_{*}$ are close to the maximum observed values for the CRSF energy. The results depicted in Fig. 2 confirm that $L_{X}>L_{\text {crit }}$ in the group 1 sources and $L_{\mathrm{X}}<L_{\text {crit }}$ in the group 2 sources. The situation is less clear for highly variable sources with luminosity $L_{X}$ that crosses over the line $L_{\mathrm{X}}=L_{\text {crit }}$, such as V $0332+53$ and $4 \mathrm{U} 0115+63$. These two sources display a negative correlation between $E_{\text {cyc }}$ and $L_{X}$ in the supercritical regime, as expected, but the trend does not reverse as predicted by our model when $L_{X}<L_{\text {crit }}$. This suggests that these sources may actually always remain supercritical, despite the fact that they cross the vertical line in Fig. 3. This behavior can be accommodated within our model by slightly changing the parameters $\Lambda$ and $w$ in Eq. (55). Or, alternatively, the behavior of these sources could indicate that their mass and radius values deviate from the canonical values assumed here.

We have developed simple physical models describing the quantitative variation of $E_{\text {cyc }}$ with $L_{X}$ in the supercritical and subcritical sources, given by Eq. (58), with the emission height $h$ set using $h=h_{\mathrm{s}}$ (Eq. (40)) for the supercritical sources (group 1), 
and $h=h_{\mathrm{c}}$ (Eq. (51)) for the subcritical sources (group 2). In Fig. 3 the formulas we derived for $E_{\text {cyc }}$ as a function of $L_{X}$ were compared with the data for the supercritical and subcritical sources. The agreement between the theoretical predictions and the data suggests that our fundamental model for the physical processes operating in these systems is essentially correct. It is important to note that our formulas for the emission heights $h_{\mathrm{c}}$ and $h_{\mathrm{s}}$ are not equipped to handle the trans-critical case with $L_{\mathrm{X}} \sim L_{\text {crit }}$, and therefore further work is required in order to treat sources such as V $0332+53$ and 4U $0115+63$.

Although the observational picture is still not complete, especially for sources with highly variable luminosities, nonetheless we believe that the emerging bimodal paradigm for the variability of the CRSF energy with luminosity in XRBPs supports the hypothesis that we are seeing direct evidence for two different accretion regimes, depending on whether the luminosity is above or below the corresponding value of $L_{\text {crit }}$ for the given surface magnetic field strength $B_{*}$. The agreement between the theoretical predictions and the observational data is rather surprising given the level of approximation employed here. We therefore believe that these ideas can provide a useful framework for future detailed modeling of XRBPs with variable luminosities, as well as motivation for further observations.

Acknowledgements. The authors gratefully acknowledge generous support provided by the ISSI in Bern, Switzerland, during the course of this work. M.T.W. acknowledges support from the US Office of Naval Research. I.C. acknowledges financial support from the French Space Agency CNES through CNRS. The authors are also grateful for assistance and useful comments from S. Müller, B. West, K. Wolfram, and A. Bodaghee. We also thank S. Tsygankov for providing observational data in digital form. Finally, we are grateful to the referee, Lev Titarchuk, for a careful reading of the manuscript and several insightful suggestions for improvement.

\section{Appendix A: Effective velocity}

The characteristic emission height in the supercritical sources treated in Sect. 3.1 is estimated by comparing the vertical transport time for the radiation with the mean escape time for the photons to diffuse out through the walls of the accretion column. This requires a determination of the "effective velocity", $v_{\text {eff }}$, which is defined as the net photon transport velocity in the vertical direction, taking into account the competing effects of advection and diffusion. The former process tends to drag photons downward toward the stellar surface, and the latter process tends to transport photons in the opposite direction, upward through the accretion column. The relationship between the effective velocity $v_{\text {eff }}$ and the flow velocity $v$ is expressed by the dimensionless parameter $\xi$, defined by

$\xi \equiv \frac{v_{\text {eff }}}{v}$.

In "trapped" regions of the flow, vertical advection and diffusion are nearly balanced, and consequently $v_{\text {eff }} \ll v$ and $\xi \ll 1$ (Becker 1998). Trapping tends to occur in the lower, hydrostatic region of the accretion column in the supercritical sources. In this situation, the photons tend to "hover" in a small altitude range until they escape through the walls of the accretion column. Hence this effect reduces the size of the emission region in the supercritical sources treated in Sect. 3.1.

The gas enters the top of the accretion column moving supersonically, but it must come to rest at the stellar surface, and therefore the flow passes through a sonic point somewhere in the column. The sonic point is located in the middle of the radiationdominated shock, where the flow begins to decelerate from the incident free-fall velocity $v_{\text {ff }}$ (Eq. (3)). Hence the sonic point represents the top of the hydrostatic sinking region, where the radiation tends to escape, and we will therefore estimate the value of $\xi$ using conditions there.

In order to determine the flow velocity at the sonic point, it is useful to consider the conservation of mass and momentum in the hydrostatic region of the column. We have

$J \equiv \rho v=$ const.,$\quad I \equiv P_{\mathrm{r}}+\rho v^{2}=$ const.

where $J$ and $I$ denote the fluxes of mass and momentum, respectively. These two fluxes are conserved in the roughly cylindrical, hydrostatic portion of the accretion column.

We can use Eqs. (A.2) to obtain a relationship between the flow velocity $v$ and the radiation Mach number, $\mathcal{M}$, defined by

$\mathcal{M} \equiv \frac{v}{a}$,

where $a$ denotes the radiation sound speed, given by

$a=\sqrt{\frac{\gamma P_{\mathrm{r}}}{\rho}}, \quad \gamma=\frac{4}{3}$.

The result obtained is

$\frac{I}{J}=v\left(1+\frac{1}{\gamma \mathcal{M}^{2}}\right)=\frac{7}{4} v_{\mathrm{c}}$,

where $v_{\mathrm{c}}$ denotes the flow velocity at the radiation sonic point, where $\mathcal{M}=1$.

The value of $\xi$ can be estimated by examining the vertical propagating of the photons in a radiation-dominated accretion column described by the exact dynamical solution obtained by Basko \& Sunyaev (1976) and Becker (1998). This solution assumes a cylindrical geometry in the hydrostatic lower region of the accretion column. The total radiation energy flux in the vertical direction is given by

$E_{r}=4 P_{\mathrm{r}} v+\frac{c}{n_{\mathrm{e}} \sigma_{\|}} \frac{\mathrm{d} P_{\mathrm{r}}}{\mathrm{d} z}$

where $P_{\mathrm{r}}$ is the radiation pressure, and the first and second terms on the right-hand side represent advection and diffusion, respectively. We define $v_{\text {eff }}$ by writing

$4 P_{\mathrm{r}} v_{\mathrm{eff}} \equiv E_{r}$,

so that $v_{\text {eff }}$ represents the "effective" bulk velocity that would yield the correct energy flux.

By combining Eqs. (A.6) and (A.7), we obtain

$v_{\mathrm{eff}}=v+\frac{c}{4 n_{\mathrm{e}} \sigma_{\|} P_{\mathrm{r}}} \frac{\mathrm{d} P_{\mathrm{r}}}{\mathrm{d} z}$

or, equivalently,

$\xi=\frac{v_{\text {eff }}}{v}=1+\frac{c m_{\mathrm{p}}}{4 \sigma_{\|} J P_{\mathrm{r}}} \frac{\mathrm{d} P_{\mathrm{r}}}{\mathrm{d} z}$,

where we have eliminated the electron number density using the relation $J=n_{\mathrm{e}} m_{\mathrm{p}} v$. The pressure $P_{\mathrm{r}}$ can be expressed in terms of the flow velocity $v$ by using Eqs. (A.2) to write

$P_{\mathrm{r}}(z)=I-J v(z)$. 
Using this relation to substitute for the pressure $P_{\mathrm{r}}$ in Eq. (A.9) yields

$\xi=1-\frac{c m_{\mathrm{p}}}{4 \sigma_{\|}(I-J v)} \frac{\mathrm{d} v}{\mathrm{~d} z}$.

The exact solution for the flow velocity profile $v(z)$ in a cylindrical accretion column is given by (Basko \& Sunyaev 1976; Becker 1998)

$v(z)=v_{\mathrm{c}}\left(\frac{14}{7+2 \epsilon_{\mathrm{c}}}\right)\left[1-\left(\frac{14}{7-2 \epsilon_{\mathrm{c}}}\right)^{-z / z_{\mathrm{st}}}\right]$,

where

$\epsilon_{\mathrm{c}}=\frac{3 m_{\mathrm{p}}^{2} c^{2}}{8 r_{0}^{2} J^{2} \sigma_{\perp} \sigma_{\|}}$

denotes the value of the dimensionless total energy flux $\left(E_{r}+\right.$ $\left.\rho v^{3} / 2\right) /\left(J v_{\mathrm{c}}^{2}\right)$ at the sonic point, and

$z_{\mathrm{st}}=r_{0}\left(\frac{8 \epsilon_{\mathrm{c}} \sigma_{\perp}}{3 \sigma_{\|}}\right)^{1 / 2}\left(\frac{2}{7+2 \epsilon_{\mathrm{c}}}\right) \ln \left(\frac{14}{7-2 \epsilon_{\mathrm{c}}}\right)$

is the altitude of the sonic point above the stellar surface.

Combining Eqs. (A.11)-(A.14), we obtain after some algebra

$\xi=1-\left(1-\frac{4 v}{7 v_{\mathrm{c}}}\right)^{-1}\left[1-\left(\frac{7+2 \epsilon_{\mathrm{c}}}{14}\right) \frac{v}{v_{\mathrm{c}}}\right]$.

In particular, at the sonic point $\left(z=z_{\mathrm{st}}\right)$, we have $v=v_{\mathrm{c}}$, and therefore our result for $\xi$ reduces to

$\left.\xi\right|_{z=z_{\mathrm{st}}}=\frac{2 \epsilon_{\mathrm{c}}-1}{6}$

The dimensionless energy flux is related to the incident Mach number of the flow, $\mathcal{M}_{\infty}$, via (Becker 1998)

$\epsilon_{\mathrm{c}}=\frac{1}{2}+\frac{3}{\mathcal{M}_{\infty}^{2}}$.

Combining Eqs. (A.16) and (A.17) yields for the value of $\xi$ at the radiation sonic point

$\left.\xi\right|_{z=z_{\text {st }}}=\frac{1}{\mathcal{M}_{\infty}^{2}}$.

We use this relation in Sect. 3.1, where we estimate the height of the emission region in the supercritical sources.

\section{Appendix B: Coulomb stopping depth}

In the subcritical sources treated in Sect. 3.2, radiation pressure is insufficient to decelerate the flow to rest at the stellar surface. In this regime, the final deceleration likely occurs via Coulomb interactions between the infalling plasma and the mound of dense gas that has built up just above the stellar surface. We can estimate the vertical extent of this region, and therefore obtain an approximation of the characteristic emission altitude in the subcritical sources, by computing the Thomson optical depth, $\tau$, measured from the stellar surface, and setting it equal to the value required to stop the flow, denoted by $\tau_{*}$. Nelson et al. (1993) carried out a detailed calculation of $\tau_{*}$, and the final result is presented in their equation Eq. (3.34). In this section we summarize the derivation.

A123, page 12 of 13
In a magnetized pulsar accretion column, the Coulomb stopping of the gas occurs via coupling between infalling protons and stationary electrons in the mound. The corresponding rate of change of the proton kinetic energy,

$E_{\mathrm{p}}=\frac{1}{2} m_{\mathrm{p}} v^{2}$

is given by Eq. (3.31) from Nelson et al. (1993), which states that

$\frac{\mathrm{d} E_{\mathrm{p}}}{\mathrm{d} z}=\frac{4 \pi n_{\mathrm{e}} e^{4}}{m_{\mathrm{e}} v^{2}} \ln \Lambda_{\mathrm{c}}$,

where $\ln \Lambda_{\mathrm{c}}$ is the Coulomb logarithm and $v$ is the velocity of the protons. Note that the right-hand side of Eq. (B.2) is positive in our sign convention since the value of $E_{\mathrm{p}}$ decreases with decreasing altitude.

Using Eq. (B.1) to substitute for $v$ yields the equivalent form

$\frac{\mathrm{d} E_{\mathrm{p}}^{2}}{\mathrm{~d} z}=\frac{m_{\mathrm{p}}}{m_{\mathrm{e}}} 4 \pi n_{\mathrm{e}} e^{4} \ln \Lambda_{\mathrm{c}}$.

We can transform from the altitude $\mathrm{d} z$ to the Thomson depth $\mathrm{d} \tau$ using

$\mathrm{d} \tau=n_{\mathrm{e}} \sigma_{\mathrm{T}} \mathrm{d} z$

which yields

$\frac{\mathrm{d} E_{\mathrm{p}}^{2}}{\mathrm{~d} \tau}=\frac{m_{\mathrm{p}}}{m_{\mathrm{e}}} \frac{4 \pi e^{4}}{\sigma_{\mathrm{T}}} \ln \Lambda_{\mathrm{c}}$.

Treating the Coulomb logarithm as a constant and integrating with respect to $\tau$, we obtain the solution

$E_{\mathrm{p}}(\tau)=E_{0}\left(1-\frac{\tau}{\tau_{*}}\right)^{1 / 2}$

where the stopping depth, $\tau_{*}$, is defined by

$\tau_{*}=\frac{m_{\mathrm{e}}}{m_{\mathrm{p}}} \frac{\sigma_{\mathrm{T}} E_{0}^{2}}{4 \pi e^{4} \ln \Lambda_{\mathrm{c}}}$,

and the incident proton kinetic energy, $E_{0}$, is equal to the free-fall value,

$E_{0}=\frac{1}{2} m_{\mathrm{p}} v_{\mathrm{ff}}^{2}$

Substituting for the Thomson cross section, $\sigma_{\mathrm{T}}$, in Eq. (B.7) using

$\sigma_{\mathrm{T}}=\frac{8 \pi e^{4}}{3 c^{4} m_{\mathrm{e}}^{2}}$,

yields the equivalent result

$\tau_{*}=\frac{1}{6 \ln \Lambda_{\mathrm{c}}} \frac{m_{\mathrm{p}}}{m_{\mathrm{e}}} \frac{v_{\mathrm{ff}}^{4}}{c^{4}}$.

In magnetized pulsar accretion columns, with discrete Landau levels, the Coulomb logarithm is given by Eq. (3.32) from Nelson et al. (1993), which states that

$\ln \Lambda_{\mathrm{c}}=\ln \left(2 n_{\max }\right)$, 
P. A. Becker et al.: Bimodal spectral variation in X-ray pulsars

where the maximum excited Landau level, $n_{\max }$, is given by

$n_{\max }=\frac{m_{\mathrm{e}} v_{\mathrm{ff}}^{2}}{2 E_{\mathrm{cyc}}}$.

Combining Eqs. (B.10) and (B.11) and substituting for $v_{\mathrm{ff}} \mathrm{using}$ Eq. (3) gives the final result,

$\tau_{*}=51.4\left(\frac{M_{*}}{1.4 M_{\odot}}\right)^{2}\left(\frac{R_{*}}{10 \mathrm{~km}}\right)^{-2} \frac{1}{\ln \left(2 n_{\max }\right)}$,

in agreement with Eq. (3.34) from Nelson et al. (1993). For typical X-ray pulsar parameters, we obtain $\tau_{*} \sim 20$, and this is the value utilized in computing the characteristic emission height in the subcritical sources in Sect. 3.2.

\section{References}

Araya-Góchez, R. A., \& Harding, A. K. 2000, ApJ, 544, 1067

Arons, J., Klein, R. I., \& Lea, S. M. 1987, ApJ, 312, 666

Basko, M. M., \& Sunyaev, R. A. 1976, MNRAS, 175, 395

Becker, P. A. 1998, ApJ, 498, 790

Becker, P. A., \& Wolff, M. T. 2007, ApJ, 654, 435

Blum, S., \& Kraus, U. 2000, ApJ, 529, 968

Burnard, D., Arons, J., \& Klein, R. 1991, ApJ, 367, 575

Caballero, I., Kretschmar, P., Santangelo, A., et al. 2007, A\&A, 465, L21

Davidson, K. 1973, Nature Phys. Sci., 246, 1

Ferrigno, C., Becker, P. A., Segreto, A., Mineo, T., \& Santangelo, A. 2009, A\&A, 498,825
Frank, J., King, A. R., \& Raine, D. J. 2002, Accretion Power in Astrophysics (Cambridge: Cambridge Univ. Press)

Giacconi, R., Gursky, H., Kellogg, E., et al. 1971, ApJ, 167, L67

Harding, A. K., Kirk, J. G., Galloway, D. J., Meszaros, P., et al. 1984, ApJ, 278, 369

Imamura, J. N., Durisen, R. H., Lamb, D. Q., \& Weast, G. J. 1987, ApJ, 313, 298

Isenberg, M., Lamb, D. Q., \& Wang, J. C. 1998, ApJ, 493, 154

Klochkov, D., Staubert, R., Santangelo, A., et al. 2011, A\&A, 532, A126

Mihara, T., Makishima, K., \& Nagase, F. 2004, ApJ, 610, 390

Mowlavi, N., Kreykenbohm, I., Shaw, S. E., et al. 2006, A\&A, 451, 187

Müller, S., Obst, M., Kreykenbohm, I., et al. 2011, The X-ray Universe 2011,

Presentations of the Conference held in Berlin, Germany

Negueruela, I., \& Okazaki, A. T. 2001, A\&A, 369, 108

Negueruela, I., Roche, P., Fabregat, J., \& Coe, M. J. 1999, MNRAS, 307, 695

Nelson, R., Salpeter, E., \& Wassermann, I. 1993, ApJ, 418, 874

Lamb, F. K., Pethick, C. J., \& Pines, D. 1973, ApJ, 184, 271

Langer, S. H., \& Rappaport, S. 1982, ApJ, 257, 733

Parkes, G. E., Murdin, P. G., \& Mason, K. O. 1980, MNRAS, 190, 537

Reynolds, A. P., Quaintrell, H., Still, M. D., et al. 1997, MNRAS, 288, 43

Shakura, N. I., \& Sunyaev, R. A. 1973, A\&A, 24, 337

Staubert, R., Shakura, N. I., Postnov, K., et al. 2007, A\&A, 465, L25

Steele, I. A., Negueruela, I., Coe, M. J., \& Roche, P. 1998, MNRAS, 297, L5

Tananbaum, H., Gursky, H., Kellogg, E. M., et al. 1972, ApJ, 174, L143

Trümper, J., Pietsch, W., Reppin, C., et al. 1978, ApJ, 219, L105

Tsygankov, S. S., Lutovinov, A. A., Churazov, E. M., \& Sunyaev, R. A. 2007, Astron. Lett., 33, 368

Tsygankov, S. S., Lutovinov, A. A., \& Serber, A. V. 2010, MNRAS, 401, 1628

Vasco, D., Klochkov, D., \& Staubert, R. 2011, A\&A, 532, A99

Wang, Y.-M., \& Frank, J. 1981, A\&A, 93, 25

Yamamoto, T., Sugizaki, M., Mihara, T., et al. 2011, PASJ, 63, 751 